\title{
Medición de la atención en un call center usando box-jenkins
}

\author{
Recibido: 16/08/12 Aceptado: 26/09/12
}

\author{
Eduardo Raffo Lecca ${ }^{1}$ \\ Luis Ráez Guevara ${ }^{2}$ \\ Carlos Quispe Atúncar ${ }^{3}$
}

\begin{abstract}
RESUMEN
El artículo revisa los conceptos de predicción y presenta una nueva metodología que utiliza la clase Box-Jenkins para la predicción de la demanda de llamadas, que efectúan los clientes a los centros de llamadas más conocidos como call-center.

El estudio concluye que el empleo de herramientas de serie de tiempos funciona de manera eficiente, lo que ha de redundar en la mejora de la eficiencia y competitividad en los call-center.
\end{abstract}

Palabras clave: predicción, series de tiempo, técnicas univariantes, modelos autorregresivos, modelos matemáticos, metodología Box-Jenkins, ARIMA.

\section{MEASUREMENT OF CARE IN A CALL- CENTER USING BOX-JENKINS}

\section{ABSTRACT}

The article reviews the concepts of prediction and presents a new methodology, which uses the BoxJenkins class, for prediction of demand for calls, which make customers call centers known as callcenter.

The study concludes that the use of time series tools, works efficiently, which would be in improving the efficiency and competitiveness in the call center.

Keywords: forecasting, time series, univariante techniques, autoregressive models, mathematical models, Box-Jenkins, ARIMA.

\section{SITUACIÓN PROBLEMÁTICA}

Con la llegada a nuestro país de las estrategias de venta por teléfono, como tiempos compartidos e incluso sistemas de emergencias médicas; como ocurrió en otros países, hace que tengamos que definir la importancia de la administración del recurso humano involucrado en estos nuevos negocios, que surgieron como miniempresas de escasa o nula inversión tecnológica, los teleoperadores.

Los centros de atención telefónica han experimentado un gran aumento en las últimas décadas, hoy en día están presentes en casi cualquier compañía que interactúe con un alto número de clientes. La capacidad está determinada principalmente por el número de operadores humanos o telefonistas.

\section{ANTECEDENTES DE LA INVESTIGACIÓN}

En la primera mitad de la década de 1990 aparecen los primeros centros de llamadas profesionales, con la presencia de agencias de telemarketing y atención de llamadas. En la segunda mitad de la década de 1990, el mercado fue depurándose, las comercializadoras fueron paulatinamente dejando su lugar a las agencias profesionales por un lado y a los centros de llamadas internas por el otro.

Algunos factores que han contribuido al auge de los centros de atención:

a) Con la llegada de la empresa Telefónica, la tecnología de telecomunicaciones y de procesamiento de datos, se desarrollaron aceleradamente a finales de la década del 90.

b) Los costos de las telecomunicaciones a nivel mundial cayeron drásticamente por razones tecnológicas y económicas, en el tiempo.

c) La inversión en telecomunicaciones de la década de 1990 ubicó a nuestro país en un nivel de avanzada, superando incluso los ratios de países más desarrollados.

d) El desempleo hizo que trabajar como teleoperador fuera una alternativa.

1 Ingeniero Industrial. Profesor del Departamento Académico de Ingeniería de Sistemas e Informática, UNMSM. E-mail: eraffolecca@yahoo.es.

2 Ingeniero Industrial. Profesor del Departamento Académico de Diseño y Tecnología Industrial, UNMSM. E-mail: 1raezg@unmsm.edu.pe, luisraez2010@hotmail.com.

3 Ingeniero Industrial. Profesor del Departamento Académico de Ingeniería de Sistemas e Informática, UNMSM. E-mail: cquispea@unmsm.edu.pe, cquispea9@gmail.com. 
En la industria de telemarketing se condensa una nueva forma laboral, el teleoperador, cuyas funciones responden a una lógica de producción en serie dentro del nuevo modelo de sociedad postindustrial que se ha denominado sociedad de la información.

Entre otros datos: la existencia de una fuerza laboral de 2.86 millones de personas en Estados Unidos (Datamonitor, 2004). En otros países, Europa cuenta con 750 mil personas operando en esta industria (Datamonitor, 2004) y en Francia hay 200 mil.

En 1970, los investigadores de la Universidad of Wisconsin-Madison George E. P. Box y Gwilym M. Jenkins, desarrollaron una metodología destinada a identificar, estimar y diagnosticar modelos dinámicos de series temporales en los que la variable tiempo juega un papel fundamental. Una parte importante de esta metodología está pensada para liberar al investigador en econometría de la tarea de especificación de los modelos, dejando que los propios datos temporales de la variable a estudiar indiquen las características de la estructura probabilística subyacente.

Los procedimientos a analizar se contraponen a la "forma tradicional" de identificar y especificar un modelo apoyados en las teorías subyacentes al fenómeno analizado aunque, convenientemente utilizados, los conceptos y procedimientos a examinar constituyen una herramienta útil para ampliar y complementar los conocimientos econométricos básicos.

Una serie temporal, conocida como serie cronológica o histórica, "es una sucesión de observaciones de una variable tomados secuencialmente en el tiempo" (Box, Jenkins y Reinsel, 2008).

Estudios recientes como "evaluación de las series temporales para la previsión de la demanda de emergencias sanitarias" de Díaz-Hierro y otros, publicado en la revista española Emergencias (2012), "Pronóstico de llamadas de emergencias sanitarias mediante modelos de series temporales", realizado en la Empresa Pública de Emergencias Sanitarias (EPES) en la ciudad de Málaga (2008), son ejemplos de investigaciones en los que se aplican las técnicas de pronósticos al estudio de las demandas de llamadas. El análisis central está centrado en el uso de la metodología ARIMA de Box-Jenkins.

\section{PREDICCIÓN}

Una de las dimensiones más sobresalientes en la que difieren las técnicas discrecionales y cuantitati- vas es su exactitud. Los datos numéricos y los métodos estadísticos pueden proporcionar respuestas precisas y objetivas, las cuales en muchas ocasiones pueden ser muy importantes. En otras, podrían no ser tan importantes. Esta diferencia plantea la cuestión de si se pueden obtener pronósticos más exactos de los métodos cuantitativos que de los métodos discrecionales.

En situaciones repetitivas donde los datos pueden recopilarse sistemáticamente, los métodos cuantitativos realmente proporcionan predicciones más exactas. Los cualitativos no procesan información de manera precisa y consistente; no obstante, es importante tener presente que tanto el juicio como los métodos cuantitativos trabajan con el mismo principio básico: la identificación de los patrones o relaciones existentes. La diferencia estriba en los métodos al preparar pronósticos.

Una de las más grandes ventajas de los métodos cuantitativos es la facilidad que se tiene para identificar los elementos de estacionalidad, tendencia, ciclicidad y aleatoriedad de manera eficiente y razonablemente objetiva. Subsecuentemente, cada uno de los tres primeros elementos, pueden extrapolarse para preparar pronósticos más exactos. Por definición, la aleatoriedad no puede pronosticarse, pero una vez que ha sido aislada, su magnitud se puede estimar y utilizar para determinar el alcance de la probable variación entre los resultados reales y pronosticados. En otras palabras, la aleatoriedad ayuda a determinar el alcance de la incertidumbre en las predicciones.

\section{LA TEORÍA DE LAS SERIES DE TIEMPO}

Durante las últimas tres décadas, se ha producido un importante incremento en el número de trabajos publicados sobre modelización y predicción de la demanda de los clientes.

Los métodos de predicción pueden ser agrupados en dos grandes categorías: métodos cualitativos y métodos cuantitativos (Bowerman, O'Connell y Koehler, 2007). En la Figura 1 podemos observar una clasificación de los principales métodos de predicción utilizados. Los primeros en aquellas situaciones en las que el pasado no proporciona información directa sobre el fenómeno considerado, como ocurre, por ejemplo, en marketing con la aparición de productos totalmente nuevos en el mercado, de los cuales no se tienen ningún tipo de referencia. 
Figura 1: Métodos de predicción

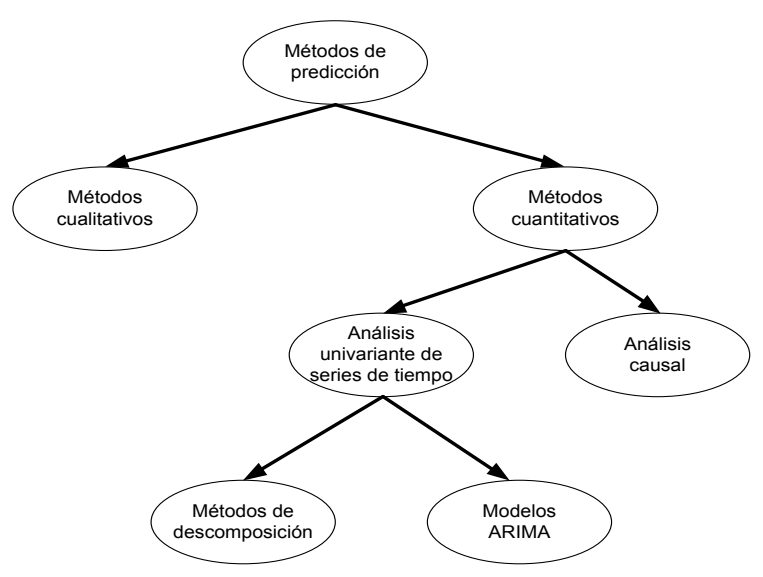

Fuente: Makridakis

En los métodos cuantitativos el objetivo es extraer toda la información posible contenida en los datos y, en base al patrón de conducta seguida en el pasado, realizar estimaciones sobre el futuro. El modelo de la serie de tiempos es quizás el más común de los modelos de predicción cuantitativo (Makridakis y Wheelwright, 2010). En relación a este tipo de métodos, se pueden considerar dos enfoques alternativos: análisis univariante de series temporales y análisis causal.

La serie de tiempo cronológica o histórica, se define en una sucesión cronológica de observaciones de una variable particular (Bowerman y otros). Una secuencia de cantidad de bienes enviados desde un proveedor o una serie de observaciones por hora dentro de un proceso químico (Box, Jenkins y Reinsel), son ejemplos de una serie cronológica. Básicamente, lo que se pretende con el estudio de las series temporales es el conocimiento de una variable a través del tiempo a partir de este conocimiento, y bajo el supuesto de que no se van a producir cambios estructurales, poder realizar predicciones. Es, por tanto, la estabilidad temporal del conjunto de factores causales que operan sobre la variable dependiente, el elemento clave sobre el que se articulan las predicciones.

En los modelos causales (econométricos) se tienen en cuenta factores externos que pueden influir en las variables objeto de estudio. Relacionadas estas variables, en un modelo que describe la relación entre estas variables y la variable que se desea pronosticar. En el análisis univariante no se necesita conocer ninguna relación de causalidad explicativa del comportamiento de la variable endógena.

Entre las técnicas univariantes existen algunas sencillas, como los modelos autorregresivos de primer orden, o los modelos de tendencia lineal o exponencial, mientras que otras técnicas resultan más complejas, (pe. los modelos Box-Jenkins o los modelos de función de transferencia).

Existen cinco áreas importantes de aplicación de los modelos de serie de tiempos:

1. El pronóstico de valores futuros.

2. La determinación de funciones de transferencias.

3. El uso de indicadores de variables de entrada en las funciones de transferencias para representar un modelo.

4. El examen de las interrelaciones entre las variables de interés y la determinación de modelos dinámicos multivariables.

5. El diseño de sistemas de control.

Los pronósticos de series de tiempo, consideran al sistema como una caja negra y no intentan descubrir los factores que afectan su comportamiento. Existen tres razones, según Makridakis, para considerar un sistema como una caja negra. La primera "puede ser que no se entienda el problema y aún si se explica, puede ser sumamente difícil medir las relaciones que se supone gobiernan su comportamiento". Una segunda razón es pronosticar qué sucederá; y la tercera empleando series de tiempo, el costo de predecir puede ser relativamente bajo.

La predicción univariante se utiliza en problemas económicos, principalmente con dos objetivos:

1. La predicción de algunas variables explicativas de un modelo causal, cuando se espera que en el futuro conserven algunas de las características de su evolución en el pasado.

2. La predicción a corto plazo, debido a su gran capacidad de recoger la dinámica en el comportamiento de la variable en estudio; además en condiciones normales, cuando no existen bruscas alteraciones con respecto a la experiencia reciente de la variable.

Entre las técnicas univariantes existen algunas sencillas, como por ejemplo los modelos autorregresivos de primer orden o los modelos de tendencia lineal o exponencial, mientras que, otras técnicas resultan más complejas, como son los modelos Box-Jenkins o los modelos de función de transferencia. "En teoría, la metodología de Box-Jenkins se puede usar para pronosticar cualquier tipo de serie de tiempo. Es la más adecuada cuando los componentes de la serie de tiempo están cambiando conforme transcurre el tiempo" (Bowermann, 2007). 


\section{MODELOS MATEMÁTICOS}

La autocorrelación es la herramienta básica utilizada en la mayoría de los métodos avanzados, tales como ARMA de Wold, ARIMA de Box-Jenkins, ARARMA de Parzen y filtrado de Kalman.

La herramienta de autocorrelación (AC) sirve para identificar el patrón básico y determinar el modelo apropiado que corresponde a la serie de datos. El coeficiente de correlación es la asociación entre dos variables y describe lo que tiende a sucederle a una de ellas si se da un cambio en la otra.

El grado de la relación varía entre -1 y +1 . Un valor aproximado a +1 implica una fuerte relación positiva entre las dos variables, un coeficiente de -1 expresa lo opuesto. Los aumentos se asociarán con una disminución, un valor de cero indica que las variables no están relacionadas.

El grado de la relación de una autocorrelación se mide mediante el coeficiente de correlación, con la excepción de que describe la asociación entre valores de la misma variable en diferentes periodos.

En la Figura 2, las variables $A$ y $B$ se consideran como dos variables diferentes y separadas, aún cuando ambas provienen del mismo conjunto de datos. La variable $B$ se construye eliminando el primer término de $A$, es una creación de variables con rezagos de 1 tiempo.

Figura 2: Variables con rezago de 1 tiempo

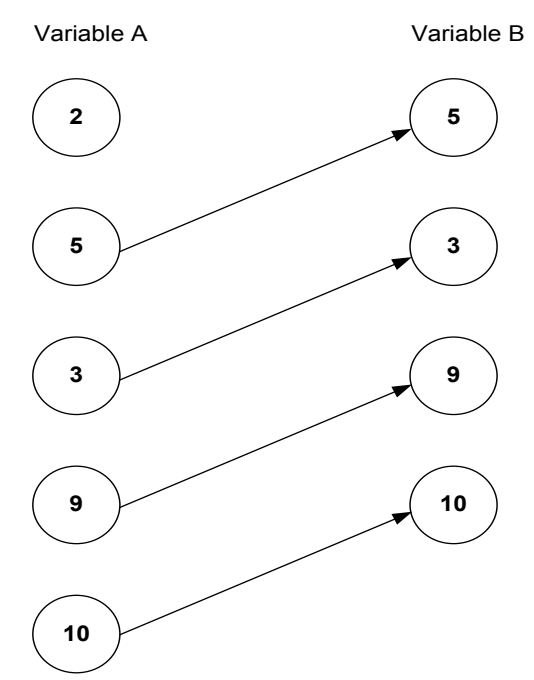

Fuente: Makridakis

La autocorrelación es una medida de asociación entre valores sucesivos de la misma variable.

Son tres grandes clases de modelos de series de tiempo que pueden describir cualquier tipo o patrón de datos de series de tiempo:

\section{Autorregresivos (AR)}

2. De promedio móvil (MA)

3. De promedio móvil autorregresivo mixto (ARMA)

Un modelo autorregresivo tiene la forma siguiente: $Y_{\mathrm{t}}=\phi_{1} Y_{\mathrm{t}-1}+\phi_{2} Y_{\mathrm{t}-2}+\cdots+\phi_{p} Y_{\mathrm{t}-p}+e_{\mathrm{t}}$

Donde $Y_{t} Y_{t}$ es la variable dependiente $\mathrm{y}$ $Y_{\mathrm{t}-1}, Y_{\mathrm{t}-2}, \cdots, Y_{\mathrm{t}-p} Y_{\mathrm{t}-1}, Y_{\mathrm{t}-2}, \cdots, Y_{\mathrm{t}-p}$ son las variables independientes. El término $e_{\mathrm{t}} e_{\mathrm{t}}$ se conoce como error o término residual, que representa las perturbaciones aleatorias que no se pueden explicar por el modelo.

Un segundo modelo es el de promedio móvil; y tiene la forma siguiente:

$Y_{\mathrm{t}}=e_{\mathrm{t}}-\theta_{1} e_{\mathrm{t}-1}-\theta_{2} e_{\mathrm{t}-2}-\cdots-\theta_{p} e_{\mathrm{t}-q}$

Donde $Y_{t} Y_{t}$ es la variable dependiente, y $e_{t-1}, e_{t-2}, \cdots, e_{t-q} e_{t-1}, e_{t-2}, \cdots, e_{t-q}$ son los valores anteriores del error. El término $e_{\mathrm{t}} e_{\mathrm{t}}$ se conoce como error o término residual, que representa las perturbaciones aleatorias que no se pueden explicar por el modelo.

Un tercer modelo es el mixto que tiene la forma siguiente:

$Y_{t}=\phi_{1} Y_{t-1}+\phi_{2} Y_{t-2}+\cdots+\phi_{p} Y_{t-p}+e_{t}-\theta_{1} e_{t-1}-\theta_{2} e_{t-2}-\cdots-\theta_{p} e_{t-q}$

Donde $Y_{\mathrm{t}} Y_{\mathrm{t}}$ es la variable dependiente, y $e_{t-1}, e_{t-2}, \cdots, e_{t-q} e_{t-1}, e_{t-2}, \cdots, e_{t-q}$ son los valores anteriores del error. El término ${ }^{\theta_{t} \theta_{t}}$ se conoce como error o término residual, que representa las perturbaciones aleatorias que no se pueden explicar por el modelo.

Una serie de tiempos $z_{1}, z_{2}, \cdots, z_{N} z_{1}, z_{2}, \cdots, z_{N}$ de $N$ sucesivas observaciones muestrales de una población infinita de tales series de tiempos; de los procesos estocásticos; más comúnmente denominados procesos.

Una importante clase de modelos estocásticos para describir series de tiempos que ha recibido gran atención, son los llamados modelos estacionarios, los que asumen que el proceso permanece en equilibrio estadístico, con una propiedad probabilística que no cambia sobre el tiempo, en particular variando un nivel promedio constante fijo y con una varianza constante. En actividades de la economía, muchas series de tiempo a menudo son representadas como no estacionarias y en particular no tienen un nivel promedio constante. Técnicas como las propuestas por Holt, Winters y Brown son apropiadas para modelos no estacionarios (Box, Jenkins y Reinsel, 2008). 
El operador de desplazamiento hacia atrás (Backward Shift Operator) $\boldsymbol{B} \boldsymbol{B}$ se define por $\boldsymbol{B} z_{t}=z_{t-1}$ $\boldsymbol{B} z_{\mathrm{t}}=z_{\mathrm{t}-1}$ y $\boldsymbol{B}^{m} z_{\mathrm{t}}=z_{\mathrm{t}-m} \boldsymbol{B}^{m} z_{\mathrm{t}}=z_{\mathrm{t}-m}$. La operación inversa es desarrollada por el operador hacia adelante (Forward Shift Operator) y viene dado por $\boldsymbol{F}=\boldsymbol{B}^{-1} \boldsymbol{F}=\boldsymbol{B}^{-1}$ y $\boldsymbol{F} z_{\mathrm{t}}=z_{\mathrm{t}+1} \boldsymbol{F} z_{\mathrm{t}}=z_{\mathrm{t}+1}$, con $F^{m} z_{t}=z_{t+m} F^{m} z_{t}=z_{t+m}$. Otro operador importante es el operador de diferencia hacia atrás ${ }^{\nabla \nabla}$, definido por $\nabla z_{t}=z_{t}-z_{t-1} \nabla z_{t}=z_{t}-z_{t-1}$; lo que permite escribir como:

$$
\nabla z_{\mathrm{t}}=z_{\mathrm{t}}-z_{\mathrm{t}-1}=(1-B) z_{\mathrm{t}}
$$

Los modelos estocásticos que se emplean se basan en observaciones de series de tiempos $z_{\mathrm{t}} z_{\mathrm{t}}$ cuyos valores con frecuencia pueden ser generados desde una serie de valores independientes $a_{t}$ $a_{t}$. Estos valores conocidos como shock provienen de una distribución fija, usualmente asumida como distribución normal con media cero y varianza $\sigma_{a}{ }^{2} \sigma_{a}{ }^{2}$. Tal secuencia de variables aleatorias $a_{t}, a_{t-1}, a_{t-2}, \cdots a_{t}, a_{t-1}, a_{t-2}, \cdots$ es llamada proceso de ruido blanco.

El proceso de ruido blanco $a_{t} a_{t}$ es transformado al proceso $z_{\mathrm{t}} z_{\mathrm{t}}$ por un filtro lineal atendiendo al operador $\psi(B) \psi(B)$ : $z_{\mathrm{t}}=\mu+a_{\mathrm{t}}+\psi_{1} a_{\mathrm{t}-1}+\psi_{2} a_{\mathrm{t}-2}+\cdots=\mu+\psi(B) a_{\mathrm{t}}$

En general $\boldsymbol{\psi}(\boldsymbol{B}) \boldsymbol{\psi}(\boldsymbol{B})$ es planteado como: $\psi(B)=1+\psi_{1} B+\psi_{2} B^{2}+\cdots$

La secuencia $\psi_{1}, \psi_{2}, \cdots \psi_{1}, \psi_{2}, \cdots$ formada por los pesos, se dice que es estable y el proceso $z_{\mathrm{t}} z_{\mathrm{t}}$ es estacionario si cumple:

$$
\sum_{j=0}^{\infty}\left|\psi_{j}\right|<\infty
$$

En otro caso el proceso $z_{\mathrm{t}} z_{\mathrm{t}}$ es no estacionario.

En un modelo autorregresivo se tiene el operador: $\phi(B) \tilde{z}_{\mathrm{t}}=a_{\mathrm{t}}$

Que es equivalente a $\tilde{z}_{t}=\phi^{-1}(B) a_{t}=\psi(B) a_{t}$

En un modelo de promedio móvil se tiene el operador: $\tilde{z}_{t}=\theta(B) a_{t}$

En un modelo ARMA se cumple: $\phi(\boldsymbol{B}) \tilde{z}_{\mathrm{t}}=\theta(\boldsymbol{B}) a_{\mathrm{t}}$

Este modelo emplea $p+q+2 p+q+2$ parámetros desconocidos: $\mu, \phi_{1}, \phi_{2}, \cdots, \phi_{p}, \theta_{1}, \theta_{2}, \cdots, \theta_{p}, \sigma_{a}{ }^{2}$

El nivel de fluctuaciones ocurre en diferentes puntos de tiempo. Tales conductas son representadas por un operador autorregresivo generalizado $\varphi(\boldsymbol{B})$
$\varphi(B)$, donde uno o más ceros de la raíz del polinomio se encuentran en un círculo unitario. Si existen d raíces unitarias y las otras raíces se encuentran fuera del círculo, el operador $\varphi(\boldsymbol{B}) \varphi(\boldsymbol{B})$ puede escribirse como: $\varphi(B)=\phi(B)(1-B)^{d}$

Donde $\phi(B) \phi(B)$ es el operador autorregresivo estacionario. Esto es un modelo que puede representar conductas no estacionarias homogéneas es de la forma: $\varphi(\boldsymbol{B}) z_{\mathrm{t}}=\phi(\boldsymbol{B})(1-\boldsymbol{B})^{d} z_{\mathrm{t}}=\theta(\boldsymbol{B}) a_{\mathrm{t}}$

Esto es: $\phi(\boldsymbol{B}) w_{\mathrm{t}}=\theta(\boldsymbol{B}) a_{\mathrm{t}}$ Donde

$$
w_{\mathrm{t}}=(1-\boldsymbol{B})^{d} z_{\mathrm{t}}=\nabla^{d} z_{\mathrm{t}}
$$

La conducta no estacionaria homogénea a menudo es representada por un modelo que llama a la désima diferenciación del proceso para ser estacionario. En la práctica d es usual entre los valores 0,1 y 2. Para el caso de $d=0$, el proceso tiene conducta estacionaria.

El proceso $\phi(\boldsymbol{B}) w_{t} \phi(\boldsymbol{B}) w_{t}$ provee un poderoso modelo para describir procesos de series de tiempos con conductas estacionarias y no estacionarias. Es conocido como modelos autorregresivos integrados de medias móviles (procede del término anglosajón Autorregresive, Integrated and Moving Average), o ARIMA de orden $(p, q, d)(p, q, d) ; y$ viene definido por:

$w_{t}=\phi_{1} w_{t-1}+\phi_{2} w_{t-2}+\cdots+\phi_{p} w_{t-p}-\theta_{1} a_{t-1}-\theta_{2} a_{t-2}-\cdots-\theta_{p} a_{t-q}$ Con $w_{\mathrm{t}}=\nabla^{d} z_{\mathrm{t}}$

El modelo ARIMA es la integración del proceso estacionario ARMA con $w_{t} w_{t}$ diferenciado $d$ veces.

\section{METODOLOGÍA BOX-JENKINS}

La metodología de los modelos ARIMA fue formalizada por Box y Jenkins en 1976, de allí que también se les denomina modelos Box-Jenkins. Este enfoque parte del hecho de que la serie temporal que se trata de predecir es generada por un proceso estocástico cuya naturaleza puede ser caracterizada mediante un modelo. Para efectuar la estimación de un modelo ARIMA se requiere de una serie temporal diaria, mensual, trimestral, etc, con un elevado número de observaciones.

La metodología Box-Jenkins proporciona una forma apropiada para modelar o encontrar modelos dinámicos para emplearlos en pronóstico y control. En la Figura 3, se presentan los 5 pasos del método, de tres etapas con la finalidad de construir modelos de series de tiempo univariables. 
Esta metodología está pensada para liberar al investigador de la tarea de buscar el modelo, los propios datos temporales de la variable indicarán la estructura probabilística subyacente. En parte, los procedimientos a analizar se contraponen a la "forma tradicional" de identificar y especificar un modelo apoyados en las teorías subyacentes al fenómeno analizado.

La principal base para el desarrollo de las series de tiempos es el supuesto del equilibrio estático. En particular el supuesto de la clase, ser estacionario. Usualmente una serie de tiempos estacionaria es descrita por la media, varianza y la función de autocorrelación.

Figura 3: Método de predicción de Box-Jenkins

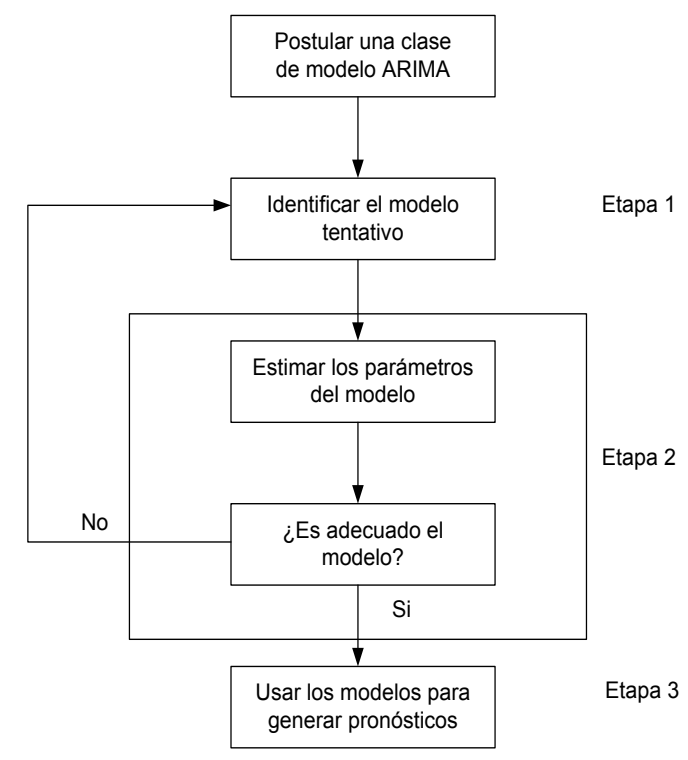

Fuente: Box-Jenkins

Las observaciones desde una serie temporal discreta en los tiempos $\tau_{1}, \tau_{2}, \cdots, \tau_{N} \tau_{1}, \tau_{2}, \cdots, \tau_{N}$ denotados por $z\left(\tau_{1}\right), z\left(\tau_{2}\right), \cdots, z\left(\tau_{N}\right) z\left(\tau_{1}\right), z\left(\tau_{2}\right), \cdots, z\left(\tau_{N}\right)$, pueden ser capturadas desde uno de dos caminos: Por muestreo desde un tiempo continuo o por acumulación de la variable sobre un periodo de tiempo.

Un proceso estocástico se dice que un proceso estrictamente estacionario, si sus propiedades son invariantes con respecto a un desplazamiento en el tiempo (variación de t). Es decir, considerando que $t_{1}, t_{2}, \cdots, t_{m} t_{1}, t_{2}, \cdots, t_{m}$ sus asociadas distribuciones conjuntas es la misma con $\mathrm{m}$ observaciones $z\left(t_{1+k}\right), z\left(t_{2+k}\right), \cdots, z\left(t_{m+k}\right)$ $z\left(t_{1+k}\right), z\left(t_{2+k}\right), \cdots, z\left(t_{m+k}\right)$, hecho en los tiempos $t_{1+k}, t_{2+k}, \cdots, t_{m+k} t_{1+k}, t_{2+k}, \cdots, t_{m+k}$. Es decir, en un proceso discreto estrictamente estacionario no está afectado por el desplazamiento de todas las observaciones hacia adelante o hacia atrás por cualquier cantidad entera $\mathrm{k}$.
La media de un proceso estocástico, estimado desde una media muestral es evaluada como:

$$
\tilde{z}=\frac{1}{N} \sum_{i=1}^{N} z_{i}
$$

La varianza ${\tilde{\sigma_{z}}}^{2}{\tilde{\sigma_{z}}}^{2}$ de un proceso estocástico, estimado desde una varianza muestral es:

$$
\tilde{\sigma}_{z}{ }^{2}=\frac{1}{N} \sum_{i=1}^{N}\left(z_{i}-\tilde{z}\right)^{2}
$$

Los coeficientes de autocorrelación y autocovarianza desde el supuesto de una asunción estacionaria, la función de distribución conjunta $p\left(t_{1}, t_{2}\right) p\left(t_{1}, t_{2}\right)$ es la misma para cualquier tiempo $t_{1}, t_{2} t_{1}, t_{2}$.

La covarianza será llamada autocovarianza en k y es definida como:

$\gamma_{k}=\operatorname{cov}\left[z_{\mathrm{t}}, z_{\mathrm{t}+k}\right]=E\left[\left(z_{\mathrm{t}}-\mu\right)\left(z_{\mathrm{t}+k}-\mu\right)\right]$

Similarmente, la autocorrelación en $k$ es:

$$
\begin{aligned}
& \rho=\frac{E\left[\left(z_{\mathrm{t}}-\mu\right)\left(z_{\mathrm{t}+k}-\mu\right)\right]}{\sqrt{E\left[\left(z_{\mathrm{t}}-\mu\right)^{2}\right] E\left[\left(z_{\mathrm{t}+k}-\mu\right)^{2}\right]}} \\
& =\frac{E\left[\left(z_{\mathrm{t}}-\mu\right)\left(z_{\mathrm{t}+k}-\mu\right)\right]}{\sigma_{z}^{2}}
\end{aligned}
$$

Para procesos estacionarios, la varianza $\sigma_{z}{ }^{2}=\gamma_{0}$ $\sigma_{z}{ }^{2}=\gamma_{0}$ es el mismo en el tiempo ${ }^{t+k t+k}$ como en el tiempo ${ }^{t t}$. Entonces, la autocorrelación en $\mathrm{k}$ es igual a la expresión:

$\rho=\frac{\gamma_{k}}{\gamma_{0}}$ que implica que $\gamma_{0}=1 \gamma_{0}=1$.

Esta definición de estacionariedad se conoce como estacionariedad en sentido estricto o fuerte y puede relajarse sustancialmente, la denominada estacionariedad en sentido amplio o débil.

Desde el concepto genérico de proceso estocástico puede decirse que una serie temporal es, en realidad, una muestra, una realización concreta con unos valores concretos de un proceso estocástico teórico y real. El análisis de series que se estudia se trata, a partir de los datos de una serie temporal, inferir las características de la estructura probabilística subyacente del verdadero proceso estocástico.

Bajo condiciones generales, todo proceso estocástico estacionario se presta a una especificación tipo AR $(p)$ y en consecuencia se expresa también como un MA (q). A continuación, se especifíca lo que se ha llamado "condiciones generales" y se examina en qué casos es posible la realización de un proceso AR o MA para representar un proceso estocástico estacionario. 
Para que un proceso estocástico estacionario admita una formulación del tipo que aquí se estudia han de cumplirse dos condiciones accesorias:

1. El proceso no debe ser anticipante (hipótesis de recursividad temporal); lo cual quiere decir que los valores de una variable en un momento t no dependerán de los que esta misma tome en $\mathrm{t}+\mathrm{k}$, siendo $\mathrm{k}$ cualquier valor superior a cero.

2. El proceso ha de ser invertible, ell supone que la correlación entre una variable y su pasado va reduciéndose a medida que nos alejamos más en el tiempo del momento para el que estamos considerando dicha correlación (proceso ergódico). La explicación intuitiva de esta situación derivaría de que si especificáramos una variable en función de ciertos coeficientes que nos determinen su correlación con los valores pasados de ella misma, los valores de dichos coeficientes deberían ser necesariamente inferiores a uno porque si no el proceso de infinitos números sería "explosivo".

La aproximación a los procesos estocásticos con modelos AR O MA está restringida, en términos generales, a aquellos procesos estocásticos que cumplan, al menos de forma débil, la restricción de estacionariedad. Cuando en la realidad, queremos inferir a partir de una serie temporal, la estructura del proceso estocástico mediante modelos AR o MA, se deben cubrir dos etapas:

1. Asegurar que la serie temporal, como muestra del proceso estocástico, es estacionaria.

2. En otro caso, transformar la serie temporal original de forma que la nueva serie transformada sí lo cumpla.

Una vez que los datos son estacionarios, se identifican $p$ y $q$ y $q$ por las observaciones de las autocorrelaciones y las autocorrelaciones parciales de los datos diferenciados.

Se observa como una regla general que cuando las autocorrelaciones se acercan exponencialmente a 0 , el modelo es AR y su orden se determina por el número de autocorrelaciones parciales que son significativamente diferentes de 0 . Si las autocorrelaciones parciales se acercan exponencialmente a 0 , el modelo es MA y su orden se determina por el número de autocorrelaciones estadísticamente significativa.

\section{DISCUSIÓN}

En el presente trabajo, se presenta la aplicación de una nueva metodología a la atención al cliente en un centro de llamadas para la resolución del problema de predicción de la demanda de llamadas. Este problema se conoce en la literatura como Call Center Forecasting (CCFP).

El problema de distribuir en la asignación de turnos del personal de atención de llamadas telefónicas, juega un rol central en la gestión de muchos sistemas de servicios; y su adecuada planificación significa en la mayoría de las veces considerables ahorros potenciales que justifican en gran medida, el uso de las técnicas de pronósticos y la investigación de operaciones, dado que se estima que el recurso humano es un costo fijo muy importante en los costos derivados en la atención al cliente.

Con una predicción de la demanda de llamadas, se consigue una óptima asignación de personal y de turnos, reduciendo los costos operativos, aumenta la calidad del servicio. Para la solución del CCFP, se han desarrollado diversos métodos y técnicas. Las series de tiempos son las que más se utilizan en estos pronósticos porque aportan soluciones aproximadas en menos tiempos de cálculo.

En el CCFP, un conjunto de operadoras ingresan en turnos comúnmente de un número fijo de horas (4), dedicadas a la recepción de las llamadas de los clientes. Son mayormente referidas a reclamos por el servicio que reciben, las que son luego pasadas a su posterior solución. La política es asumir un pequeño porcentaje de llamadas no atendidas. Se busca el patrón de la demanda de las llamadas de los clientes por hora, con la finalidad de pronosticar la demanda futura las llamadas. El uso final de la predicción es derivado a la asignación óptima del recurso humano en la atención al cliente.

La característica de las llamadas de los clientes, en primera instancia, es la tasa de arribos de la llamada. Esta cantidad que significa la tasa de llegadas de llamadas en la unidad de tiempo, ocurre en un proceso aleatorio que es estudiada como una serie de tiempos. Existe en cada hora del día, una conducta de la tasa de llamadas, en las primeras horas del día esta tasa es pequeña y en el transcurso del día esta tasa crece. El cliente ocasiona que la demanda de llamadas sea una caminata al azar o aleatoria.

El proceso de la recopilación de los datos se ha basado en 2065 muestras de llamadas ocurridas en periodos de cada de $1 / 2$ hora. Identificándose la cantidad de llamadas en cada uno de los periodos o ACD cantidad de demanda de llamadas (el término ACD proviene de distribuidor automático de llamadas). Ver la Figura 4 donde se presenta el patrón que representan las 2065 observaciones. 
Figura 4: Patrón de los datos

ACD

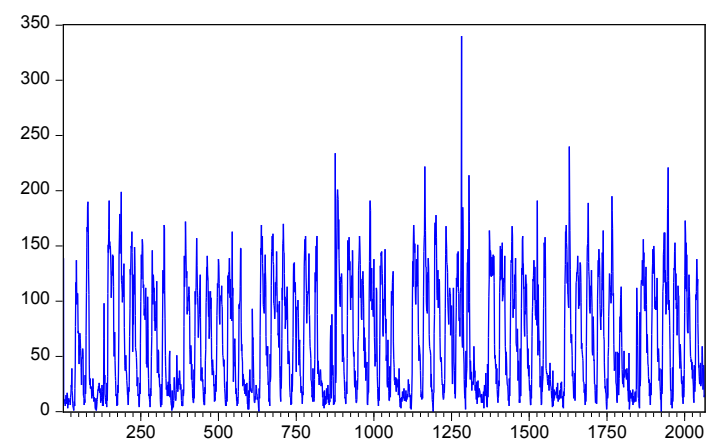

Se observa datos estadísticos recopilados en dos meses de observación:

Media $=64.90$ Mediana $=51$ Máximo $=340$ Mínimo $=0$ Desviación estándar $=50.38$

Estos datos han sido obtenidos de la Figura 5 y en la Figura 6 se presenta el diagrama de cuartiles.

Figura 5: Histograma del patrón de los datos

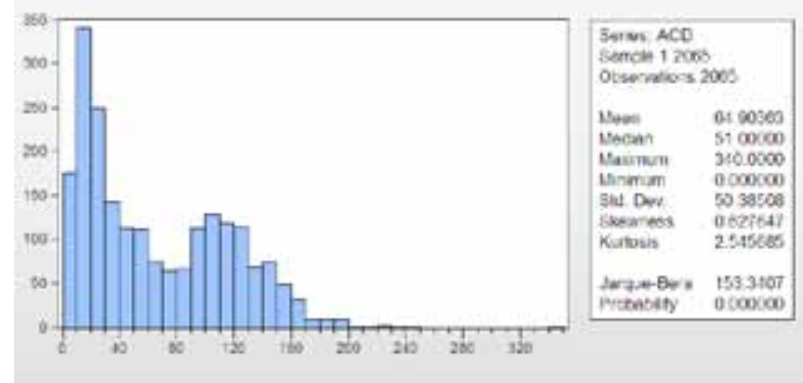

Figura 6: Diagrama de cuartiles para los datos

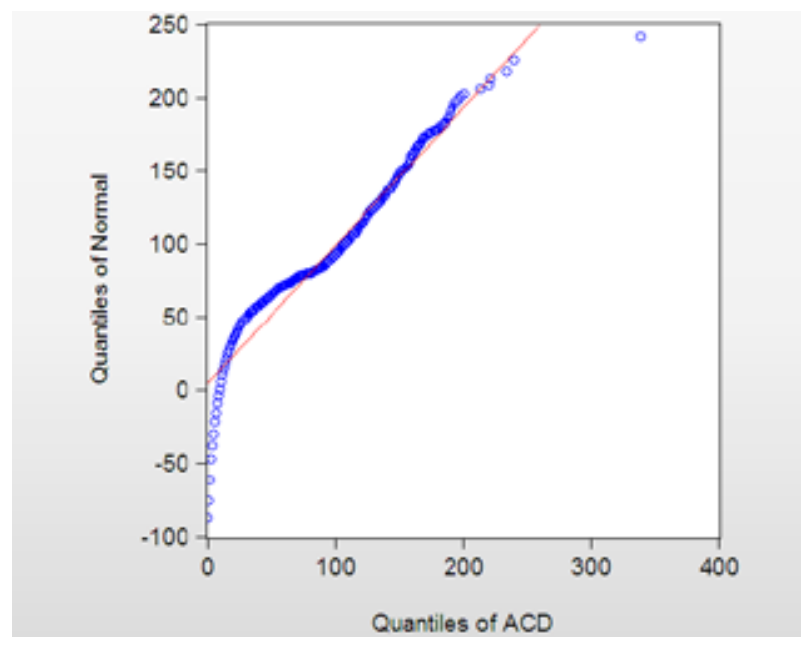

Los modelos clásicos de Box-Jenkins describen series temporales estacionarias. Para identificar en forma tentativa un modelo de Box-Jenkins es necesario determinar primero si la serie temporal a pronosticar es estacionaria. Si no es así, entonces se transforma la serie temporal en una serie de valores, para tener una serie temporal estacionaria. Una serie temporal es estacionaria si las propiedades estadísticas, la media y la variancia son esencialmente constantes a través del tiempo.

Figura 7: Diagrama de primeras diferencias $D(A C D)$

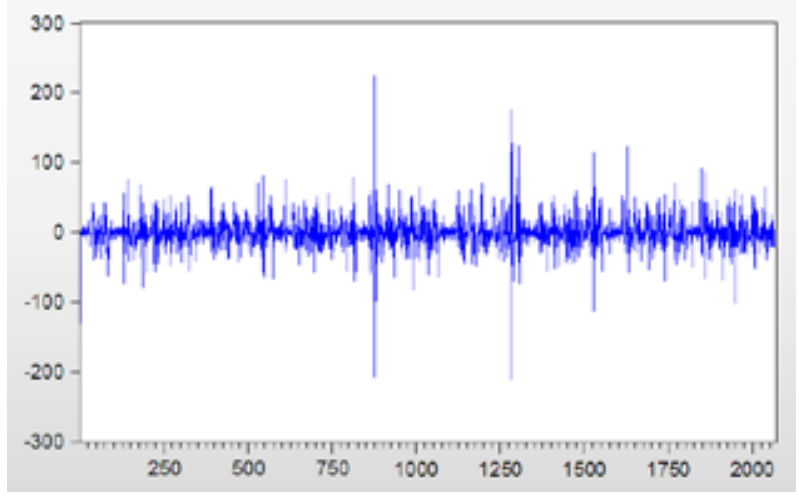

Se usa una gráfica de estos valores (en función del tiempo) para determinar si es estacionaria la serie temporal. Si al parecer los $\mathrm{n}$ valores fluctúan con variación constante respecto a una media constante $\mu$, entonces, es razonable pensar que la serie temporal es estacionaria.

Algunas veces, se transforma la serie temporal no estacionaria, en valores de una serie temporal estacionaria obteniendo las primeras y segundas diferencias de los valores de la serie temporal no estacionaria. En la Figura 7 el diagrama de primeras diferencias indican que es estacionario.

Figura 8: Correlograma

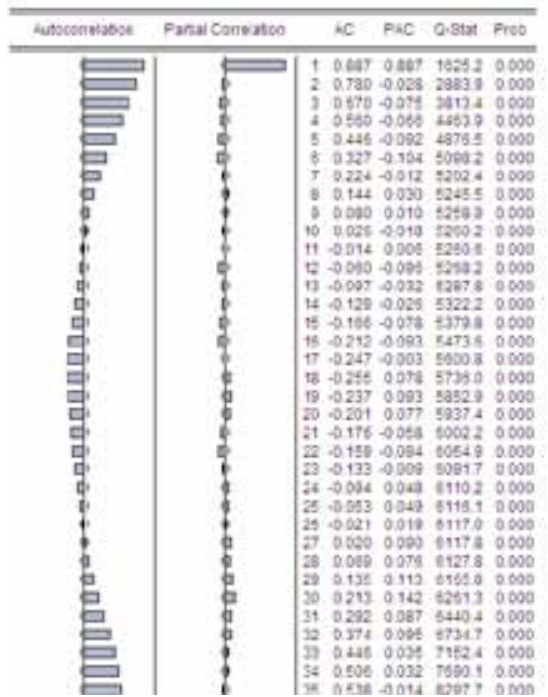


La función de autocorrelación (AC) es una lista o una gráfica de las autocorrelaciones en los desfasamientos $k=1,2, \ldots$, los correlogramas.

Al examinar la AC de la Figura 8 se observa que no se trunca o se corta, lo que significa que existe una espiga en el desfasamiento $k$, si no al contrario, se extingue (decrece en forma permanente) en seno amortiguado (una de las tres formas de extinción: forma exponencial, forma de seno o una combinación de ambas).

La experiencia indica que si la $A C$ se extingue con lentitud extrema, los valores de la serie temporal original se consideran no estacionarios. En tal caso es necesario hacer uso de una transformación de datos. Si la AC de estos datos se cortan claramente con rapidez o si se extinguen rápidamente, se debe considerar que los datos son estacionarios (Bowerman, O'Connell, 2007).

En la Figura 9, el correlograma de las primeras diferencias presenta que la $\mathrm{AC}$ se extingue; entonces los datos son estacionales.

Figura 9: Correlograma con primera diferencia

\begin{tabular}{|c|c|c|c|c|c|c|}
\hline Autocorrelation & Partial Correlation & & $A C$ & PAC & Q-Stat & Prob \\
\hline it & i & 1 & -0.023 & -0.023 & 1.0565 & $0.30 \mathrm{~s}$ \\
\hline 1 & 4 & 2 & 0.015 & 0.014 & 1.5018 & 0.472 \\
\hline$\phi$ & t & & -0.000 & 0.000 & 1.5021 & 0.682 \\
\hline ip & q & 4 & 0.021 & 0.021 & 2.3815 & $0.66 \mathrm{~s}$ \\
\hline if & if & & 0.021 & 0.022 & 3.3329 & 0.849 \\
\hline d & , & & -0.072 & .0 .072 & 14204 & 0.027 \\
\hline d. & d & & $=0.100$ & 0.105 & 35087 & 0.000 \\
\hline . & d. & & $=0.071$ & 0.078 & 45.581 & 0.000 \\
\hline , & 1 & 9 & $=0.042$ & 0.045 & 49.252 & 0.000 \\
\hline d & d & 10 & -0.067 & -0.067 & 50.647 & 0.000 \\
\hline 1) & 1 & 11 & 0.030 & 0.034 & 60.501 & 0.000 \\
\hline$y$ & f & 12 & -0.041 & -0.035 & 64.030 & 0.000 \\
\hline$y$ & d & 13 & -0.025 & -0.040 & 65.350 & 0.000 \\
\hline ) & t) & 14 & 0.030 & 0.012 & 67.234 & 0.000 \\
\hline 9 & 4 & 15 & 0.035 & 0.017 & 69.776 & 0.000 \\
\hline di & $\phi$ & 16 & -0.048 & -0.074 & 74.817 & 0.000 \\
\hline 4 & 간 & 17 & -0.121 & -0.144 & 105.18 & 0.000 \\
\hline 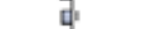 & ב & 18 & -0.114 & -0.143 & 132.06 & 0.000 \\
\hline 7 & H. & 19 & -0.077 & .0 .117 & 14436 & 0.000 \\
\hline c & ) & 20 & 0.048 & 0.021 & 148.79 & 0.000 \\
\hline 9 & d & 21 & 0.035 & 0.051 & 151.39 & 0.000 \\
\hline d. & 1 & 22 & $=0.039$ & .0 .038 & 15450 & 0.000 \\
\hline & d & 23 & -0.053 & 0.089 & 180.38 & 0.000 \\
\hline$d$ & di & 24 & -0.013 & -0.005 & 160.69 & 0.000 \\
\hline ) & dr & 25 & 0.041 & -0.050 & 164.17 & 0.000 \\
\hline 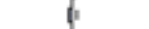 & C, & 26 & -0.030 & -0.116 & 167.19 & 0.000 \\
\hline 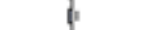 & g, & 27 & -0.041 & -0.099 & 170.73 & 0.000 \\
\hline 4 & ב, & 28 & -0.070 & -0.120 & 100.91 & 0.000 \\
\hline d & ב. & 28 & -0.058 & -0.151 & 188.23 & 0.000 \\
\hline 1 & 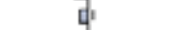 & 30 & -0.002 & -0.092 & 188.24 & 0.000 \\
\hline t & 4 & 31 & -0.016 & -0.086 & 188.78 & 0.000 \\
\hline d & 7 & 32 & 0.047 & -0.032 & 193.52 & 0.000 \\
\hline of & d & 33 & 0.052 & -0.028 & 198.21 & 0.000 \\
\hline (2) & 1) & 34 & 0.128 & 0.017 & 232.30 & 0.000 \\
\hline 曰 & 口 & 35 & 0.259 & 0.159 & 373.55 & 0.000 \\
\hline
\end{tabular}

En resumen, se presentan tres alternativas para la elección del modelo Box-Jenkins:

1. Modelo ARIMA(35, 35, 1) ARIMA $(35,35,1)$

2. Modelo ARIMA $(35,0,1) \operatorname{ARIMA}(35,0,1)$

3. Modelo incluyendo fluctuaciones estacional - SEASONAL o SEASONAL
Los resultados del modelo AR (1), MA (1) con primera diferencia se presentan en la Figura 4.10, de allí se obtiene que los coeficientes son:

$\mathrm{c}=0.002080, \mathrm{AR}(1)=-0.028776, \mathrm{MA}(1)=0.00676$

Este modelo es el ARMA $(35,35)$ con la primera diferenciación, donde se plantea desde el hecho de la repetición de las costumbres de llamadas que se repite cada 24 horas. Desde el punto de vista de ARMA, es AR (35), MA (35) en primera diferenciación equivale a un modelo autorregresivo con $p=35 p=35$ y promedio móvil con $q=35 q=35$.

Figura 10: Resultados de ARIMA $(35,35,1)$

\begin{tabular}{|c|c|c|c|c|}
\hline \multicolumn{5}{|c|}{$\begin{array}{l}\text { Dependent Variable: } D(A C D) \\
\text { Method: Least Squares } \\
\text { Date: } 11 / 27 / 11 \text { Time: } 11: 23 \\
\text { Sample (adjusted): } 32065 \\
\text { Included observations: } 2063 \text { after adjustments } \\
\text { Convergence achieved after } 7 \text { iterations } \\
\text { MA Backcast: } 2\end{array}$} \\
\hline Variable & Coefficient & Std. Error & t-Statistic & Prob. \\
\hline $\begin{array}{c}C \\
A R(1) \\
M A(1)\end{array}$ & $\begin{array}{r}0.002080 \\
-0.028776 \\
0.006176\end{array}$ & $\begin{array}{l}0.511830 \\
0.177020 \\
0.178411\end{array}$ & $\begin{array}{r}0.004064 \\
-0.162560 \\
0.034615\end{array}$ & $\begin{array}{l}0.9968 \\
0.8709 \\
0.9724\end{array}$ \\
\hline $\begin{array}{l}\text { R-squared } \\
\text { Adjusted R-squared } \\
\text { S.E. of regression } \\
\text { Sum squared resid } \\
\text { Log likelihood } \\
\text { F-statistic } \\
\text { Prob(F-statistic) }\end{array}$ & $\begin{array}{r}0.000519 \\
-0.000451 \\
23.76443 \\
1163381 \\
-9461.745 \\
0.535260 \\
0.585599\end{array}$ & \multicolumn{2}{|c|}{$\begin{array}{l}\text { Mean dependent var } \\
\text { S.D. dependent var } \\
\text { Akaike info criterion } \\
\text { Schwarz criterion } \\
\text { Hannan-Quinn criter. } \\
\text { Durbin-Watson stat }\end{array}$} & $\begin{array}{l}0.003878 \\
23.75908 \\
9.175710 \\
9.183900 \\
9.178713 \\
1.999813\end{array}$ \\
\hline $\begin{array}{l}\text { Inverted AR Roots } \\
\text { Inverted MA Roots }\end{array}$ & $\begin{array}{l}-.03 \\
-.01\end{array}$ & & & \\
\hline
\end{tabular}

El operador para la $\mathrm{n}$ diferencia de la serie $\mathrm{xx}$ se muestra a continuación: $d(x, n)=(1-B)^{n} x$

Desde la figura 11, se encuentra que:

$\mathrm{c}=0.272640, \mathrm{AR}(35)=-0.961387, \mathrm{MA}(1)=-0.841780$

Figura 11: Ecuación para ARIMA $(35,0,1)$

\begin{tabular}{|c|c|c|c|c|}
\hline \multicolumn{5}{|c|}{$\begin{array}{l}\text { Dependent Variable: D(ACD) } \\
\text { Wethod. Least Squares } \\
\text { Date: } 11 / 27 / 11 \text { Time: } 15: 49 \\
\text { Sample (adjusted): } 37 \text { 2065 } \\
\text { Included observations: } 2029 \text { after ad, ustments } \\
\text { Convergence achieved atter } 3 \text { ferabons }\end{array}$} \\
\hline Variable & Coefficient & Sid. Error & 1.Statistic & Prob: \\
\hline$\stackrel{C}{C}$ & $\begin{array}{l}0.026940 \\
0.262094\end{array}$ & $\begin{array}{l}0.895194 \\
0.021375\end{array}$ & $\begin{array}{l}0.038751 \\
12.25147\end{array}$ & $\begin{array}{l}0.8691 \\
0.0000\end{array}$ \\
\hline $\begin{array}{l}\text { R-squared } \\
\text { Adjusted R-squared } \\
\text { S.E. of regression } \\
\text { Sum squared resid } \\
\text { Log likelinood } \\
\text { F-statistic } \\
\text { Prob(F-statistic) }\end{array}$ & $\begin{array}{l}0.089049 \\
0.058590 \\
23.10707 \\
1082290 \\
.9249 .367 \\
150.3435 \\
0.000000\end{array}$ & $\begin{array}{l}\text { Mean depen } \\
\text { SD depend } \\
\text { Acaice info C } \\
\text { Schwarz critt } \\
\text { Hannan-Quit } \\
\text { Durbin-Wats }\end{array}$ & $\begin{array}{l}\text { ent vat } \\
\text { ht var } \\
\text { enon } \\
\text { on } \\
\text { critec. } \\
\text { stat }\end{array}$ & $\begin{array}{l}0.002957 \\
2394277 \\
9.119140 \\
9.124575 \\
9.121171 \\
2.217038\end{array}$ \\
\hline
\end{tabular}


El $R$ cuadrado es del orden de 0.164361 . Este modelo es el ARMA $(35,0)$ con la primera diferenciación, donde se plantea desde el hecho de la repetición de las costumbres de llamadas que se repite cada 24 horas, sin considerar el promedio móvil. Desde el punto de vista de ARMA es AR (35), MA (0) en primera diferenciación equivale a un modelo autorregresivo con $\mathrm{p}=35 \mathrm{p}=35$ y de promedio móvil con $q=0 q=0$. El R cuadrado es del orden de 0.069049, según la Figura 4.12.

Box-Jenkins, recomienda para procesos estacionales o de temporada se utilicen los términos SAR y SMA para tratar las fluctuaciones estacionales de los meses, días, horas, etc. Así se habla de SAR (p) SMA (q). El R cuadrado es del orden de 0.824989, según la Figura 12.

Figura 12: Ecuación para SAR y SMA

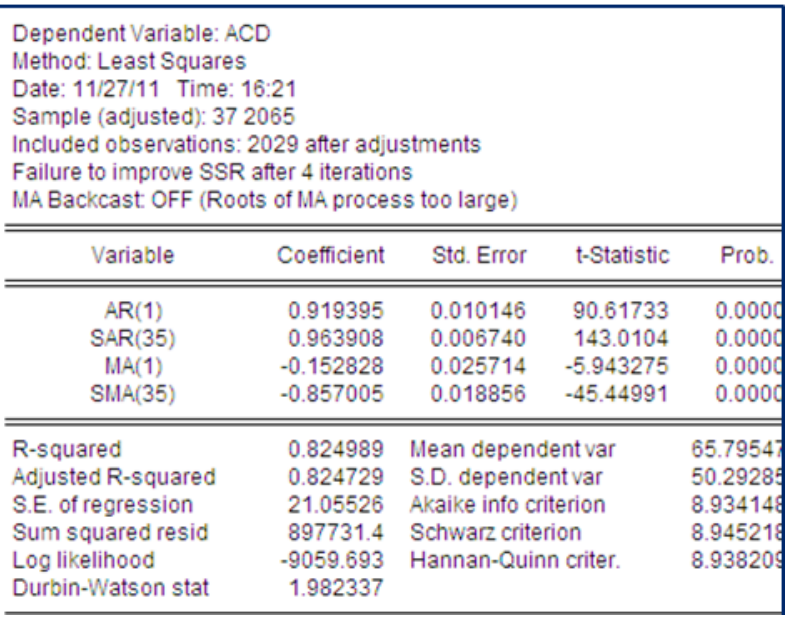

Es necesario comentar que el estadístico DurbinWatson o DW mide la correlación de la serie y un valor menor a 2 indica que existe una evidencia que la correlación de la serie es positiva. Por otro lado, el criterio de información de Akaike o AIC indica la preferencia de pequeños valores para la selección de los desplazamientos. El criterio de Schwarz o SC que es una alternativa a AIC, y es utilizado para penalizar adicionales coeficientes. El criterio HannanQuinn o $\mathrm{HQ}$ es otra alternativa de penalizaciones para los coeficientes el p-value o Prob(F-statistic) siempre es menor que el nivel de significancia.

De la Tabla 1 se concluye que el modelo SAR y SMA es el que mejor pasa la prueba de los criterios analizados poseyendo el R2 más alto. Observe que es el único que pasa la prueba DW.
Tabla 1: Resumen de los modelos planteados

\begin{tabular}{|l|c|c|c|c|c|}
\hline \multicolumn{1}{|c|}{ Modelo } & DW & AIC & SC & HQ & $\mathbf{R}^{2}$ \\
\hline ARIMA $(35,35,1)$ & 2.345 & 9.020 & 9.015 & 9.015 & 0.1644 \\
\hline ARIMA $(35,0,1)$ & 2.217 & 9.124 & 9.121 & 9.121 & 0.0690 \\
\hline SAR y SMA & 1.982 & 8.934 & 8.945 & 8.938 & 0.8249 \\
\hline
\end{tabular}

\section{CONCLUSIONES}

1) Empleando la metodología propuesta en la presente investigación, se creó un modelo de diseño de pronóstico para la demanda de llamadas telefónicas en un centro de atención de llamadas, en la ciudad de Lima.

2) Se ha realizado análisis de Box-Jenkins para Ios modelos: ARIMA $(35,35,1) \operatorname{ARIMA}(35,35,1)$ , ARIMA $(35,0,1)$ ARIMA $(35,0,1)$ y Modelos con fluctuaciones estacionales.

3) Usando el modelo Box-Jenkins para procesos estacionales o de temporada se ha encontrado que SAR y SMA son los más adecuados para predecir la demanda de llamadas del centro de llamadas en el estudio.

\section{REFERENCIAS BIBLIOGRÁFICAS}

1. BOWERMAN, B., O'CONNELL, R., KOEHLER, A. (2007). Pronósticos, series de tiempo y regresión un enfoque aplicado. México: Cengage Learning, Editores, s.a.

2. BOX, G., JENKINS, G., REINSEL, G. (2008). "Time Series Analysis: Forecasting and Control". New Jersey: John Wiley \& sons, $4^{\text {a }}$ ed.

3. DATAMONITOR (2004). "Call center outsourcing in latin america and the caribbean to 2008".

4. MAKRIDAKIS, S., WHEELWRIGTH, S. (2010). "Métodos de pronósticos". méxico: editorial Limusa, s.a.

5. Pindyck, R., Rubinfeld, R. (2001). "Colocar nombres y apellidos", McGraw-Hill. México. 